SESSION V : Innovation en génie civil - génie côtier

\title{
Pieux sous charges latérales : étude de l'effet de groupe
}

\author{
D. Remaud, J. Garnier \\ Laboratoire Central des Ponts et Chaussées(LCPC), Nantes, France \\ R. Frank \\ CERMES (ENPC-LCPC), Paris, France
}

RESUME : Afin d'étudier l'effet de groupe sur des pieux chargés latéralement, des essais sur modèles réduits de couples de pieux ont été réalisés avec la centrifugeuse du Laboratoire Central des Ponts et Chaussées. Une méthode a été établie pour obtenir les courbes de réaction $P-y$ à partir des résultats expérimentaux. On compare ensuite ces courbes à des codes de calcul reconnus : Fascicule 62 (France), Port and Harbour Research Institute (Japon) et l'American Petroleum Institute (U.S.A). Les déplacements en tête et les profils de moments fléchissants obtenus permettent de déterminer l'effet de groupe. On établit finalement des coefficients de réduction sur la réaction latérale du sol.

\section{Introduction}

Avec la maitrise des campagnes géotechniques et l'amélioration des techniques de conception, les fondations sur pieux verticaux sont devenues des solutions courantes pour reprendre les efforts horizontaux.

Le comportement d'un pieu isolé chargé latéralement a été le sujet de nombreuses recherches tant théoriques qu'expérimentales. Elles ont mené à la généralisation des méthodes dites au module de réaction dans lesquelles le sol est modélisé par des séries de ressorts horizontaux non linéaires. Ces méthodes utilisant les courbes $P$-y ( $P$ étant la réaction du sol et $y$ le déplacement horizontal du pieu) ont été intégrées dans plusieurs codes de conception d'ouvrages : Fascicule 62 (M.E.L.T., 1993), P.H.R.I. (1980), A.P.I. (1993).

En pratique cependant, les pieux sont en groupe : piles de pont, murs de quais ou fondations de piate-forme offshore. Dans ces cas, l'interaction pieu-solpieu est difficile à établir à cause de la réponse complexe et non linéaire du sol à des charges latérales.

L'interaction est en effet tridimensionnelle et de nombreux paramètres doivent être pris en compte pour établir une loi de comportement. Les modèles théoriques et numériques sont inadaptés pour une telle recherche. Etant donné le nombre d'essais nécessaires, les essais in-situ sur pieux en grandeur réelle ne sont pas non plus envisageables.

Ainsi la modélisation en centrifugeuse est une approche pratique pour l'étude d'un tel problème. Des travaux sont en cours au Laboratoire Central des Ponts et Chaussées pour étudier l'effet de groupe. Seuls les résultats relatifs à des essais sur des paires de pieux sont présentés dans cet article. 


\section{Dispositif expérimental et programme d'essais}

Garnier (1990) rappelle les conditions de similitude entre un ouvrage en grandeur réelle et son modèle en échelle réduite. La centrifugeuse du L.C.P.C. est également décrite dans cette communication. $\left.1 / 40^{\text {ème }}\right)$.

Les essais de la présente étude ont été réalisés à $40 \mathrm{~g}$ (modèles réduits au

Les pieux modèles sont conçus à partir de tubes d'aluminium de diamètre extérieur $B=18 \mathrm{~mm}$, d'épaisseur $e=1,5 \mathrm{~mm}$ et de longueur totale $L=380 \mathrm{~mm}$. La fiche en sol est de $300 \mathrm{~mm}$. Ainsi ils simulent des pieux prototypes de $720 \mathrm{~mm}$ de diamètre avec une fiche de $12 \mathrm{~m}$ : ce sont des pieux longs et flexibles $\left(E I=514 \mathrm{MN} \cdot \mathrm{m}^{2}\right)$. Ils sont équipés de 20 paires de jauges, placées tous les $15 \mathrm{~mm}$ sur sa longueur en fiche. La première paire est située à la surface du sol, la dernière à la profondeur de $285 \mathrm{~mm}$ ( $11,4 \mathrm{~m}$ en prototype).

Le matériau utilisé pour le massif de soi expérimental est un sable fin de Fontainebleau. Avec les dimensions précédemment définies, le rapport entre le diamètre des pieux et la taille des grains est environ de 900 , ce qui est très supérieur à la valeur minimale de 40 généralement acceptée comme limite pour les effets de taille.

Les pieux sont mis en place à $1 \mathrm{~g}$. Le massif de sol est reconstitué par pluviation sèche de sable autour des pieux suspendus dans le conteneur de dimensions : $L=1200 \mathrm{~mm}, l=800 \mathrm{~mm}, h=360 \mathrm{~mm}$. La pluviation est réalisée au moyen d'une trémie automatique conçue au L.C.P.C. ; elle produit un massif de sol très homogène avec une densité contrôlée (moins de $1 \%$ de déviation). Le poids volumique obtenu est dans le cas présent de $16,3 \mathrm{kN} / \mathrm{m}^{3}\left(I_{D}\right.$ de 0,89$)$.

\begin{tabular}{ccccc}
\hline $\begin{array}{c}\gamma_{\mathrm{s}} \\
\left(\mathrm{kN} / \mathrm{m}^{3}\right)\end{array}$ & $\begin{array}{c}\gamma_{\mathrm{d} \min } \\
\left(\mathrm{kN} / \mathrm{m}^{3}\right)\end{array}$ & $\mathrm{e}_{\max }$ & $\begin{array}{c}\gamma_{\mathrm{d} \max } \\
\left(\mathrm{kN} / \mathrm{m}^{3}\right)\end{array}$ & $\mathrm{e}_{\min }$ \\
\hline 26,44 & 13,64 & 0,940 & 16,83 & 0,616 \\
\hline
\end{tabular}

Tableau 1 - Propriétés du sable de Fontainebleau

Cette procédure simule une installation in-situ de pieux forés, sans refoulement du sol. L'étude se limite au cas de pieux forés, pour lesquels on peut penser que l'effet du placement à $1 \mathrm{~g}$ sur le comportement latéral du pieu est limité.

Avec le dispositif expérimental embarqué (Figures 1 et 2), différentes configurations expérimentales sont testées : pieu isolé ou couple de pieux chargé en ligne avec des entre-axes $a$ de 2B, $4 \mathrm{~B}$ et $6 \mathrm{~B}$, la tête des pieux restant libre.

\section{Interprétation}

Dans les paragraphes suivants, toutes les données sont exprimées en grandeurs prototypes. 


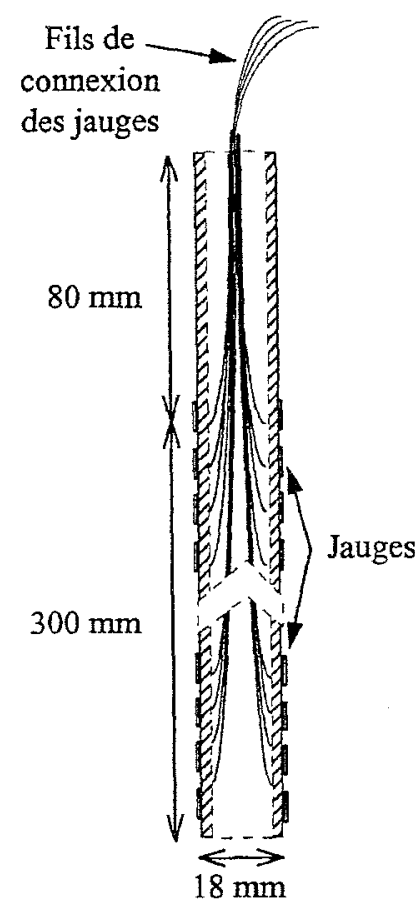

Figure 1 - Pieu modèle

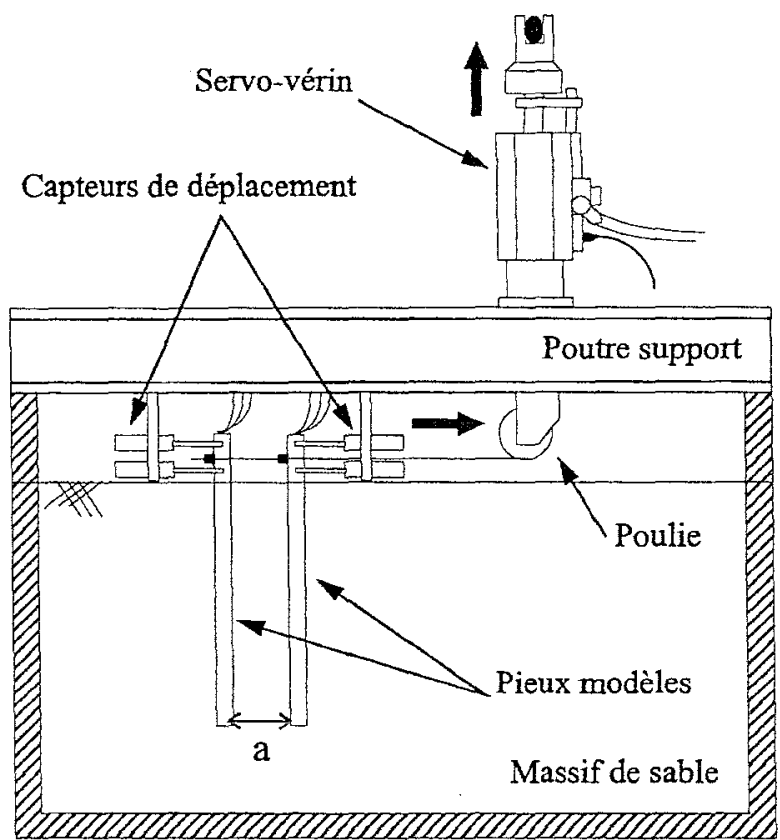

Figure 2 - Schéma du dispositif expérimental Exemple d'un conteneur de sable avec deux pieux

\subsection{Méthode}

Les jauges de déformation donnent les profils de moments fléchissants au cours du chargement latéral. L'étude est basée sur l'interprétation de ces mesures de moment.

Pour un effort latéral donné, la mesure de la distribution du moment fléchissant $M$, suivant la profondeur $z$, est utilisée pour obtenir le profil des réactions du sol $P$, par double dérivation avec le logiciel SLIVALIC5 (Degny, 1985), et le profil des déplacements du pieu $y$, par double intégration, d'après :

$$
P=\frac{d^{2} M}{d z^{2}} \text { et } y=\iint \frac{M}{E I} d z
$$

Il n'est pas possible d'obtenir de mesure de moment en pied $(z=12 \mathrm{~m})$. Le pieu étant long et flexible, on suppose que ce moment est nul. Cette valeur est ajoutée aux données expérimentales pour optimiser la procédure de double dérivation. Pour la double intégration un polynôme de degré 7 est utilisé, les constantes sont déterminées à partir du déplacement mesuré en tête et du déplacement nul en pied.

\subsection{Validation des courbes $\mathbf{P}-\mathbf{y}$}

\subsubsection{Calcul PILATE-LCPC}

L'étude étant basée sur l'obtention de courbes $P-y$, il est important de valider la procédure d'interprétation précédemment définie. Dans ce but, le logiciel PILATE-LCPC est utilisé pour vérifier la cohérence des courbes $P-y$ 
calculées. Ce logiciel calcule la réponse d'un pieu sous chargement latéral. Les courbes $P-y$ obtenues de la présente analyse sont ainsi introduites dans le logiciel.

Dans l'exemple relaté ci-dessous, le sol est modélisé par 19 couches d'épaisseurs constantes et le pieu par 20 tronçons de même caractéristiques géométriques et mécaniques. Quatre incréments de charge $F_{H}$ sont appliqués à la tête du pieu $(163 \mathrm{kN}, 322 \mathrm{kN}, 489 \mathrm{kN}$ et $652 \mathrm{kN})$.

Les résultats sont présentés en termes de déplacements et réactions du sol (Figure 3). On montre la bonne correspondance entre ces données calculées et celles déduites de l'expérience.
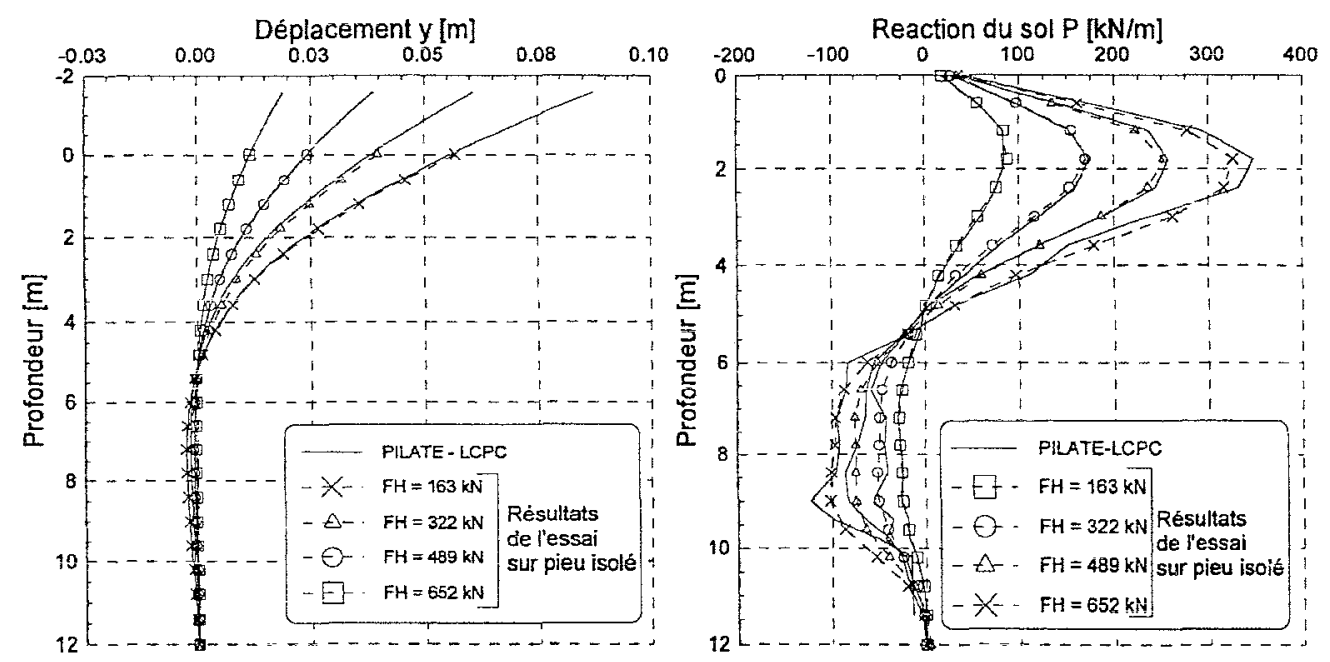

Figure 3 - Vạlidation des courbes $P$-y - Profils des déplacements du pieu et des réactions du sol

\subsubsection{Comparaison avec différents codes de calcul}

Les courbes $P$-y expérimentales étant mathématiquement validées, on les compare avec celles qui sont proposées par plusieurs codes de calcul reconnus :

- les courbes $P-y$ du Fascicule 62 nécessitent des données pressiométriques. Des essais pénétrométriques sont effectués en cours de centrifugation. Les caractéristiques pressiométriques nécessaires sont déduites de la résistance de pointe $q_{\mathrm{c}}$ par corrélations (Cassan, 1978) :

$$
p_{1}=q_{\mathrm{c}} / 9, E_{\mathrm{M}}=10 . p_{1} \text { et } p_{\mathrm{f}}=p_{\mathrm{l}} / 1,8
$$

où $E_{\mathrm{M}}$ est le module pressiométrique, $p_{1}$ et $p_{\mathrm{f}}$ sont respectivement les pressions limites et de fluage ;

- les courbes de l'A.P.I. sont calculées à partir du poids volumique $\gamma=16,3 \mathrm{kN} / \mathrm{m}^{3}$ et de l'angle de frottement interne $\varphi=40^{\circ}$;

- les courbes du P.H.R.I. sont basées sur un module de réaction, pour le sable, $k_{\mathrm{S}}=\sqrt{0,8 / \mathrm{B}}$ et $p=k_{\mathrm{S}} \cdot z \cdot y^{0,5}$ ( $B$ en mètre, $k_{\mathrm{S}}$ en $\mathrm{MN} / \mathrm{m}^{3,5}$ ).

On compare ces courbes (Figure 4 ) en deux profondeurs: $z_{1}=0,6 \mathrm{~m}$ près de la surface, et $z_{2}=2,4 \mathrm{~m}$ où les moments fléchissants sont maxima.

Pour ces deux profondeurs, les courbes $P_{-y}$ mesurées sont en accord avec les prévisions du P.H.R.I. Pour les deux autres méthodes, des différences 
importantes sont observées avec les résultats expérimentaux : ces codes tendent à sous-estimer la pression du sol près de la surface et à la surestimer en profondeur.
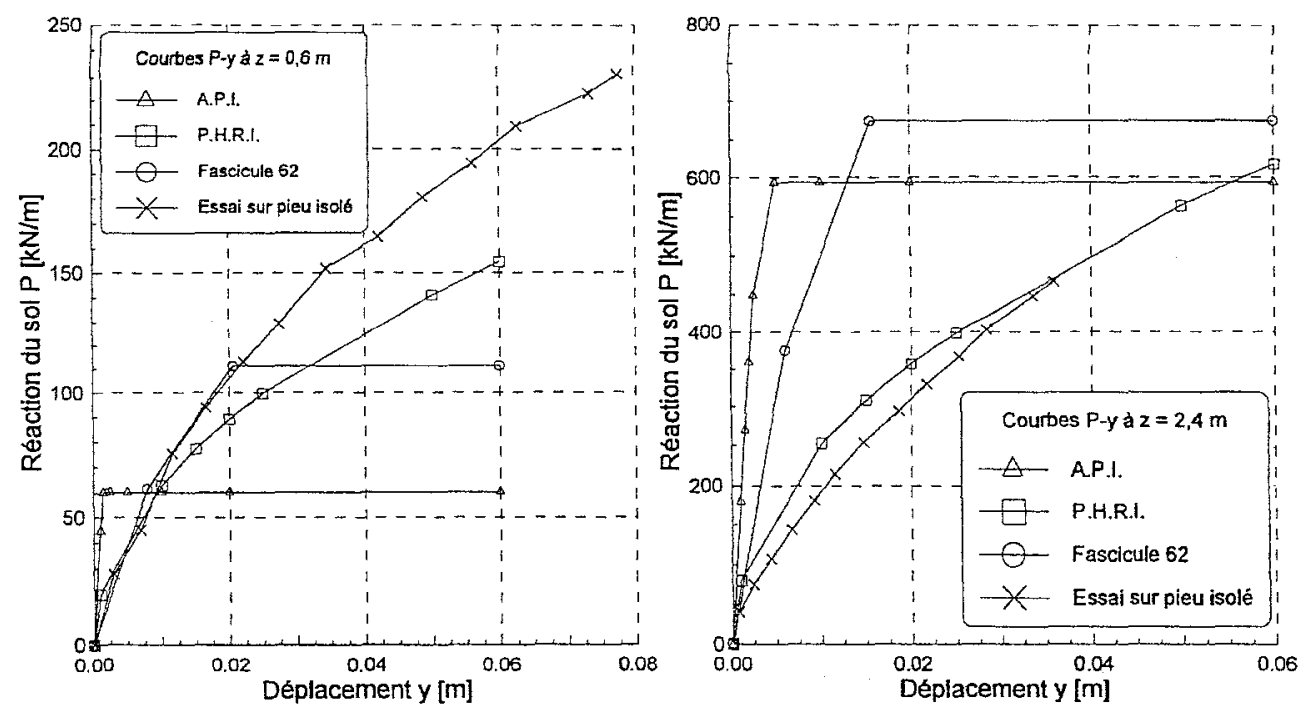

Figure 4 - Confrontation entre courbes $P$-y expérimentales et prédites

\section{L'effet de groupe}

L'entre-axe $a$ entre pieux est le facteur principal de l'effet de groupe. La réaction du sol sur les pieux arrière est réduite par rapport à celle du pieu avant en raison d'un effet d'ombre.

\subsection{Efficacité du groupe}

Comme les pieux sont libres en tête, ils peuvent ne pas avoir le même déplacement au niveau du sol. Afin d'étudier leur réponse à un effort latéral, oñ compare les déplacements de chaque pieu mesurés à la cote du point de chargement.

L'efficacité du groupe est un moyen pour quantifier l'effet de groupe. Elle est calculée pour les différentes configurations et pour le même déplacement au niveau du point de chargement comme suit :

$$
\mathrm{e}=\frac{\text { charge totale sur le groupe pour y donné }}{\mathrm{n} \times \text { charge sur un pieu isolé pour y identique }}
$$

On a obtenu $0,81,0,87$ et 0,95 respectivement pour des entre-axes de $2 B$, $4 \mathrm{~B}$ et $6 \mathrm{~B}$. On montre ainsi que les interactions dans le groupe réduisent la charge reprise pour un déplacement donné.

La distribution des efforts dans le groupe est déterminée à partir des moments calculés au niveau du sol. Le pieu avant reprend $59 \%$ de l'effort $F_{H}$ appliqué si l'entre-axe est $2 \mathrm{~B}, 56 \%$ pour $4 \mathrm{~B}$ et $51 \%$ pour $6 \mathrm{~B}$.

Ces conclusions sont cohérentes avec celles de Barton (1982) et Mezazigh (1995). Les essais montrent aussi que l'effet de groupe n'est plus sensible à partir 
d'un entre-axe dans le groupe supérieur à $6 \mathrm{~B}$.

\subsection{Moments fléchissants}

Les profils de moments fléchissants des 4 configurations testées sont tracés (Figure 5) pour le même déplacement en tête du pieu d'environ $10 \%$ du diamètre.
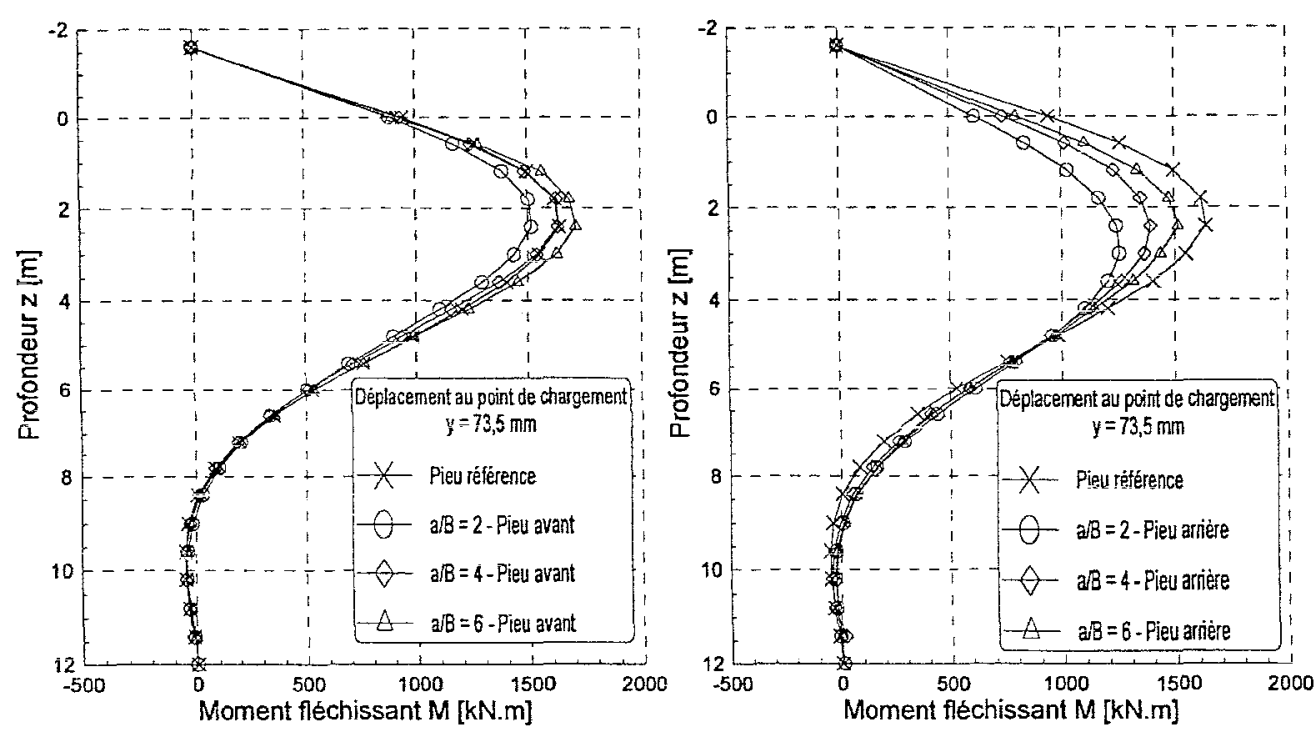

Figure 5 - Profils de moments fléchissants dans le pieu avant et dans le pieu arrière

Les moments dans le pieu avant sont très proches de ceux du pieu isolé. Dans le pieu arrière au contraire, les moments sont plus faibles que ceux du pieu isolé. Ils tendent à se rapprocher du pieu isolé avec l'augmentation de l'entre-axe des pieux du groupe.

Ainsi, les moments fléchissants maxima pour un entre-axe de $2 \mathrm{~B}$ sont de 1,5 MN.m dans le pieu avant et de 1,25 MN.m dans le pieu arrière.

L'étude des moments fléchissants confirme aussi que l'effet de groupe est négligeable à partir d'un entre-axe $a$ supérieur à $6 \mathrm{~B}$.

\subsection{Courbes $\mathbf{P}-\mathbf{y}$}

Les courbes $P$-y sont calculées avec la procédure validée $\S 2.2$. On les trace pour les deux profondeurs $z_{1}=0,6 \mathrm{~m}$ et $z_{2}=2,4 \mathrm{~m}$

Les courbes de réaction des pieux avant sont assez proches de celle du pieu isolé (Figure 6) avec une dispersion de $\pm 10 \%$. On confirme le résultat précédemment énoncé : l'effet de groupe n'est pas significatif sur le pieu avant.

Les courbes $P-y$ des pieux arrières sont tracées sur la Figure 7. Comme on l'a mis en évidence auparavant, l'effet de groupe est plus significatif sur les pieux arrières où la réaction du sol est réduite par rapport au pieu isolé et au pieu avant.

Des coefficients multiplicateurs $P_{\mathrm{m}}$ ont été proposés pour représenter l'effet de groupe sur les courbes $P-y$ (voir, par exemple, Brown et Shic, 1990) :

$$
P_{\text {pieu du groupe }}=P_{\mathrm{m}} \times P_{\text {pieu isolé }}
$$


On a mené dans ce sens une première interprétation quantitative. Le coefficient $P_{\mathrm{m}}$ a été calculé pour le pieu arrière du couple de pieux à quatre profondeurs différentes de $z_{1}=0,6 \mathrm{~m}$ à $z_{2}=2,4 \mathrm{~m}$. Les valeurs moyennes de $P_{\mathrm{m}}$ observées pour le déplacement maximum sont alors :
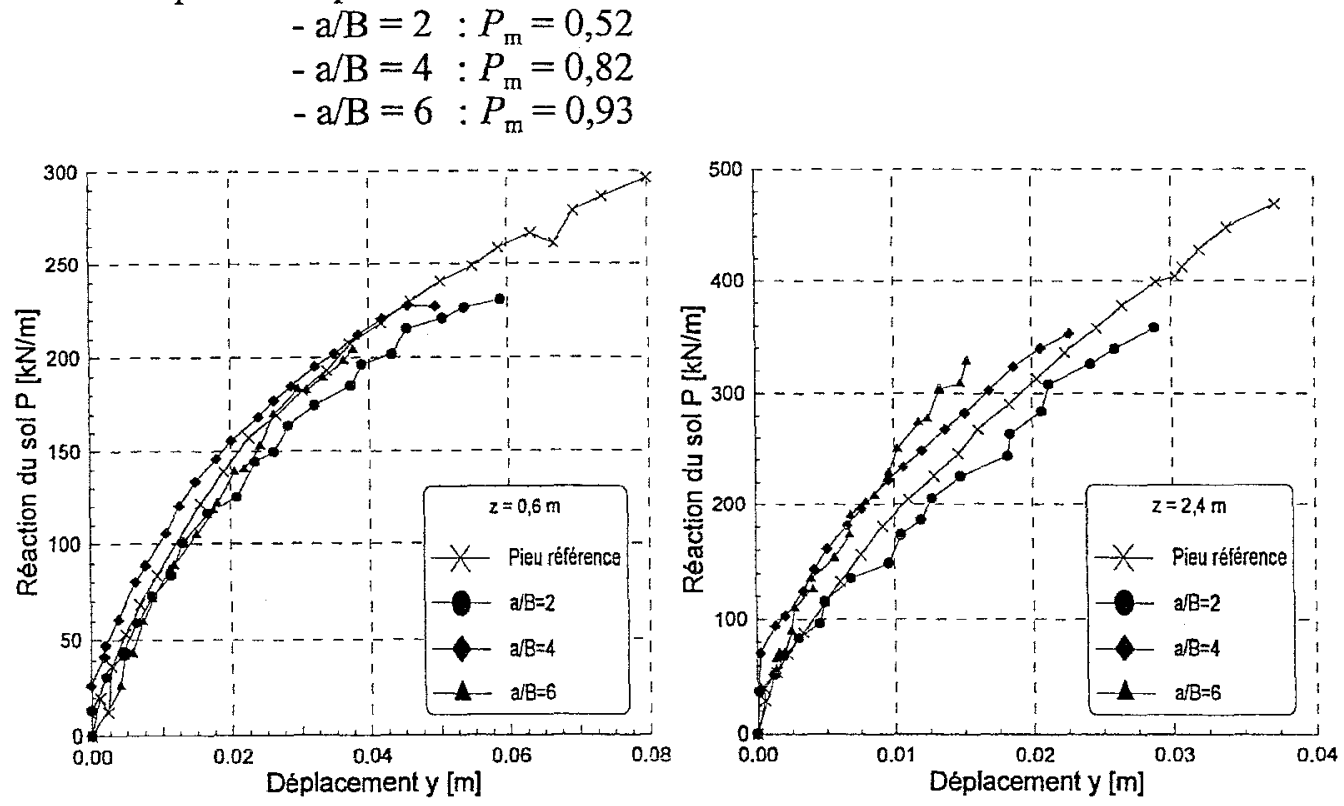

Figure 6 - Courbes de réaction $P-y$ du pieu avant
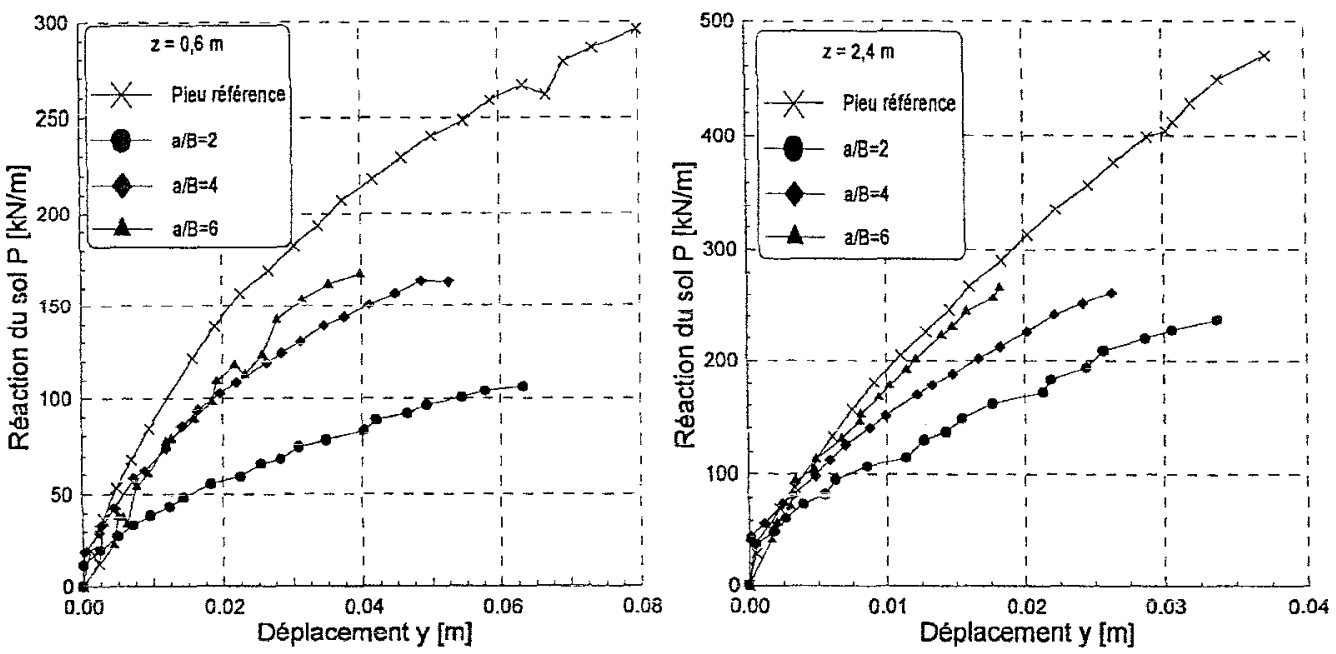

Figure 7 - Courbes de réaction $P-y$ du pieu arrière

\section{CONCLUSIONS}

On a réalisé des essais centrifugés sur des modèles de paires de pieux forés. L'effet de l'entre-axe entre les pieux a été plus particulièrement étudié.

Les courbes de réaction $P-y$ ont été déduites à partir des profils de moments fléchissants expérimentaux. Pour un pieu isolé, les courbes $P-y$ expérimentales sont comparables à celles prédites par la méthode du P.H.R.I. Des différences plus importantes sont observées avec le Fascicule 62 et l'A.P.I. 
On a mis en évidence un effet de groupe significatif pour des entre-axes $a$ entre pieux inférieurs ou égaux à $6 \mathrm{~B}$ :

- à déplacement identique, l'effort total repris par le groupe comparé à l'effort appliqué au pieu isolé est diminué (d'environ $20 \%$ pour un entreaxe de $2 \mathrm{~B}$ ) ;

- les efforts et moments fléchissants sur le pieu arrière sont réduits. Pour un entre-axe de $2 \mathrm{~B}$, le pieu arrière supporte $40 \%$ de l'effort total et le moment maximum est inférieur à celui du pieu isolé d'environ $20 \%$;

- les courbes $P-y$ sont également affectées par l'effet de groupe. Pour un entre-axe de $2 \mathrm{~B}$, la réaction du sol mobilisée sur le pieu arrière ne représente que environ $50 \%$ de celle du pieu isolé.

De plus, la réponse du pieu avant apparaît proche de celle du pieu isolé, tant en termes de moments fléchissants qu'en termes de courbes de réaction $P-y$.

Cette étude sera poursuivie par des essais portant sur des groupes de 3 pieux. On étudiera différentes dispositions (en ligne, en rang ou en trièdre) et différents entre-axes, afin de généraliser et compléter les résultats déjà obtenus.

\section{Références}

American Petroleum Institute (A.P.I.) 1993. A.P.I. RP 2A-LRFD - Section G : Foundation Design, A.P.I., pp. 64-77.

Barton, Y. O. 1982. Laterally Loaded Piles in Sand: Centrifuge Tests and Finite Element Analysis, PhD, University of Cambridge, $141 \mathrm{p}$.

Bouafia A.1990, Modélisation des pieux chargés latéralement en centrifugeuse, Thèse de Doctorat, Université de Nantes, $267 \mathrm{p}$.

Brown, D. A. \& C.-F. Shie 1990. Numerical Experiments into Group Effect on the Response of Piles to Lateral Loading, Computers and Geotechnics, $\mathrm{N}^{\circ} 10, \mathrm{pp}$. 211-230.

Cassan, M. 1978. Les essais in situ en Mécanique des Sols - Tome 1 : Réalisation et Interprétation, Ed. Eyrolles, Paris, $458 \mathrm{p}$.

Degny, E. 1985. SLIVALIC5 - Notice d'utilisation, Ed. LCPC.

Frank, R. \& J.-C. Romagny 1990. PILATE-LCPC Version 10.4 - Notice d'utilisation, Ed. LCPC.

Garnier, J. 1990. Etudes sur Modèles Réduits Centrifugés en Génie Civil, Premières Journées Génie Civil - Génie Côtier, Le Havre, pp. 101-119.

Ministère de l'Equipement, du Logement et des Transports (M.E.L.T.) 1993. Règles Techniques de Conception et de Calcul des Fondations et des Ouvrages de Génie Civil, Fascicule 62 - Titre V, Ed. M.E.L.T., France

Mezazigh, S. 1995. Etude Expérimentale de Pieux Chargés Latéralement : Proximité d'un Talus et Effet de Groupe. Thèse de Doctorat, Université de Nantes, $272 \mathrm{p}$.

Port and Harbour Research Institute (P.H.R.I.) (1980). Technical Standards for Port and Harbour Facilities in Japan, Japan Ministry of Transport, 317 p.

Remaud, D., J. Garnier \& R. Frank (à paraître) Laterally Loaded Piles in Dense Sand : Group Effects, Proceedings of Centrifuge'98, 23-25 September 1998, Tokyo, Japan. 